\title{
Comment on "Absence of a Dissipative Quantum Phase Transition in Josephson Junctions"
}

\author{
Pertti J. Hakonen $\oplus^{1,2, *}$ and Edouard B. Sonin $\oplus^{3, \dagger}$ \\ ${ }^{1}$ QTF Centre of Excellence, Department of Applied Physics, Aalto University, 00076 Aalto, Finland \\ ${ }^{2}$ Low Temperature Laboratory, Department of Applied Physics, Aalto University, 00076 Aalto, Finland \\ ${ }^{3}$ Racah Institute of Physics, Hebrew University of Jerusalem, Givat Ram, Jerusalem 9190401, Israel
}

(Received 29 May 2020; accepted 27 January 2021; published 12 March 2021)

DOI: 10.1103/PhysRevX.11.018001

Recently, Murani et al. [1] questioned the existence of the dissipative quantum phase transition (DQPT) in a single Josephson junction (JJ) predicted nearly 40 years ago [2,3]. This conclusion is based on misperception of the principles underlying the DQPT theory.

First, we recall in a nutshell the basics of the DQPT theory. The DQPT is a joint effect of Coulomb interaction, dissipation, and quantum mechanics. The Coulomb blockade makes the $\mathrm{JJ}$ an insulator at small bias. However, it is effective only if the Coulomb energy $E_{C} \sim e^{2} / C$ exceeds the quantum-mechanical uncertainty $\hbar / \tau$, where $\tau=R_{S} C$ is the time of the charge relaxation in the circuit. Here, $C$ is the capacitance of the JJ, and $R_{s}$ is the shunt resistance. The condition $E_{C} \sim \hbar / \tau$ agrees with the condition $R_{s}=R_{q}$, where the DQPT was predicted [2,3] (the dashed line in Fig. 1). Here, $R_{q}=h / 4 e^{2}$ is the quantum resistance. The existence of the DQPT has been supported by experimental work [4-9], and possible applications for qubits have been discussed [10].

The Hamiltonian of the JJ is specified by two conjugate variables, the phase $\varphi$ and the charge $Q=(\hbar C / 2 e) d \varphi / d t$ :

$$
H=\frac{Q^{2}}{2 C}+E_{J}(1-\cos \varphi)-\frac{\hbar \varphi}{2 e} I,
$$

where $I$ is the current bias. The classical equation of motion for the phase of the $\mathrm{JJ}$ is

$$
C \frac{\hbar}{2 e} \frac{d^{2} \varphi}{d t^{2}}+I_{c} \sin \varphi-I=0
$$

where $I_{c}=2 e E_{J} / \hbar$ is the critical current. Equation (2) has a solution at zero current bias $I$ with nonzero voltage $V=(\hbar / 2 e) d \varphi / d t$. The solution describes the insulating

\footnotetext{
"pertti.hakonen@aalto.fi

sonin@cc.huji.ac.il
}

Published by the American Physical Society under the terms of the Creative Commons Attribution 4.0 International license. Further distribution of this work must maintain attribution to the author(s) and the published article's title, journal citation, and DOI.
Subject Areas: Superconductivity

state. At $I<I_{c}$, there is also a stationary solution with constant $\varphi$ corresponding to the superconducting state.

In the quantum theory, the phase and charge variables become operators, and the quantum tunneling between minima of the $\varphi$-dependent washboard potential facilitates phase slips, which delocalize the phase and suppress superconductivity. The quantum tunneling leads to the formation of Bloch bands with states described by the Bloch functions $\psi(\varphi)=\Psi_{0}(\varphi) e^{i \tilde{Q} \varphi / 2 e}$. Here, $\Psi_{0}(\varphi)=$ $\Psi_{0}(\varphi+2 \pi)$ is a periodic function of $\varphi$, and $\tilde{Q}$ is the quasicharge. We consider only the lowest Bloch band with states described by the effective Hamiltonian

$$
H_{0}=E_{0}(\tilde{Q})-\frac{\hbar \varphi}{2 e} I
$$

where $E_{0}(\tilde{Q})$ is a periodic function with period $2 e$. At small $\tilde{Q}, E_{0}=\left(\tilde{Q}^{2} / 2 C^{*}\right)$, where $C^{*}$ is the effective capacitance.

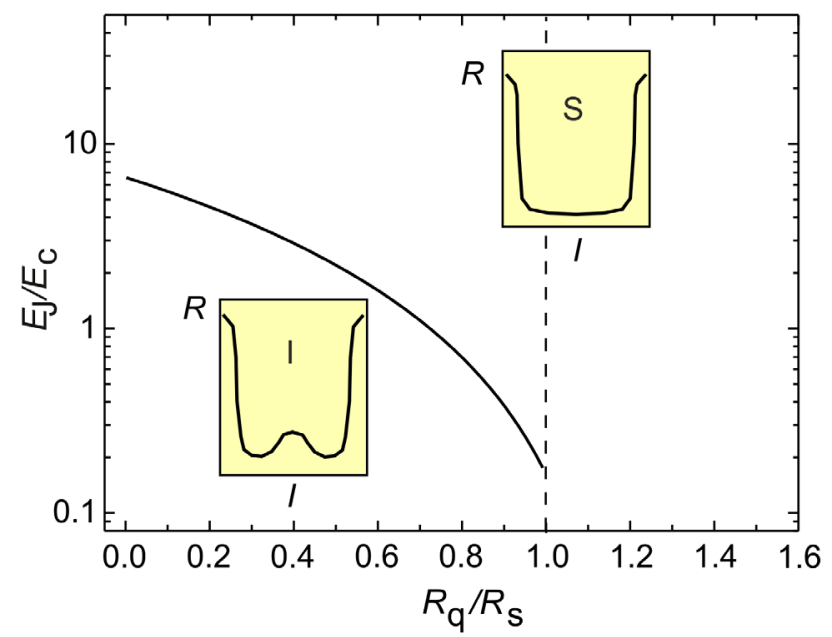

FIG. 1. The phase diagram of the JJ. The dashed line shows the DQPT of Schmid [2] and Bulgadaev [3]. The solid curve is the experimentally expected crossover curve shifted either due to voltage measurement error [4] or due to thermal fluctuations [11]. The insets show schematic shapes of $R I$ curves in the superconducting $(S)$ and the insulating $(I)$ states. 
The semiclassical equations of motion for a wave packet with average charge $\tilde{Q}$ and phase $\varphi$ are

$\frac{d \varphi}{d t}=\frac{2 e}{\hbar} \frac{\partial E_{0}}{\partial \tilde{Q}}, \quad \frac{d \tilde{Q}}{d t}=-\frac{2 e}{\hbar} \frac{\partial E_{0}}{\partial \varphi}-\frac{V}{R_{s}}=I-\frac{V}{R_{s}}$.

Without shunt $\left(R_{s} \rightarrow \infty\right)$ and bias $(I=0)$, the packet moves in the phase space with the group velocity $(2 e / \hbar) \partial E_{0} / \partial \tilde{Q}$, and we have the insulating state with the voltage drop $\partial E_{0} / \partial \tilde{Q}$ across the JJ. A current bias, however small, accelerates the packet until it reaches the Brillouin zone boundary and jumps to the opposite border of the Brillouin zone. At any such umklapp event, a Cooper pair tunnels through the JJ. In this regime of Bloch oscillations, the phase oscillates around some position without moving away from this position. Thus, the phase is localized, and the resistance of the JJ vanishes [12].

At finite $R_{s}$, there is a stationary state with constant quasicharge and voltage given by Ohm's law $V=R_{s} I$. This result means that the whole current passes through the shunt, and the junction itself is an ideal insulator. However, the group velocity in the Bloch band is restricted from above, and steady motion of the wave packet becomes impossible once the voltage reaches the threshold $V_{b}=$ $\max \left(\partial E_{0} / \partial \tilde{Q}\right)$ for electrical breakdown of the insulator. At $V>V_{b}$, Bloch oscillations start. But, in contrast to the unshunted case, the phase does not just oscillate but also steadily moves with finite average velocity. Thus, the JJ becomes a conductor with nonlinear resistance proportional to $1 / I R_{s}$ [13]. The Coulomb-blockade bump in resistance is the smoking gun of the insulating state.

The equations of motion (4) can also be employed to obtain linear ac mobility in the phase space:

$$
\mu=\frac{d I(\omega)}{d V(\omega)}=\frac{1}{R_{s}}+i \omega C^{*} .
$$

The pioneering works on the DQPT are not the last word in the theory. Schön and Zaikin [14] point out that at high ratio $E_{J} / E_{C}$ observation of the DQPT on the vertical line requires an exponentially long observation time. According to Penttilä et al. [4], experimental detection of the Coulomb-blockade bump is impossible when the breakdown voltage $V_{b}$ becomes of the order of the voltage error bar $\delta V_{m}$. Their experiment detects the bump on the line determined by the condition $\delta V_{m} \sim V_{b}$ (the solid line in Fig. 1). A similar shift of the DQPT is predicted for finite temperatures [11]. At the transition, the derivative $d R / d I$ on experimental curves changes sign, and Penttilä et al. [4] suggest the condition $d R / d I=0$ as an experimental criterion for the DQPT. This criterion requires measurements of nonlinear response.

If one increases measurement accuracy (or lowers the temperature), the effective transition line moves closer to the Schmid-Bulgadaev line. Thus, the vertical
Schmid-Bulgadaev line is an idealized asymptotic limit, which remains experimentally unattainable in practice.

Murani et al. [1] conclude that the JJ can never be an insulator, as their model involves an inductive contribution $\propto 1 / i \omega$ to Eq. (5) for the ac mobility. This model follows the suggestion of Joyez [15] that the impedance of the environment includes also the induction of the JJ itself. Hence, the inductive channel short circuits the JJ, and the insulating state is impossible. However, this suggestion contradicts the letter and the spirit of the Bloch band theory, which transforms the phase-dependent Hamiltonian Eq. (1) to the phase-independent Hamiltonian Eq. (3). Thus, the JJ inductance vanishes due to suppression of superconductivity by phase slips ignored by Murani et al. [1].

Murani et al. [1] argue that in the JJ theory one should use the compact phase determined in the interval $2 \pi$ but not the extended phase determined from $-\infty$ to $\infty$. Murani et al. [1] are free to use the compact phase, if they have a procedure to keep track of the rotation angle multiples of $2 \pi$. They do not have it, because they do not distinguish states with values of $\varphi$ differing by $2 \pi$. However, these states differ by the magnetic flux through the closed circuit [13]. The $2 \pi$ jumps ignored by Murani et al. [1] are nothing else than phase slips which are the only mechanism of the supercurrent decay, not only in JJ but also in any superfluid or superconductor.

A widely accepted concept in the theory of the $\mathrm{JJ}$ (and in condensed matter physics, in general) is the duality principle for phase and charge. According to this principle, the roles of current and voltage, as well as of resistance and conductance, are mutually exchanged. As a result, phase slips destroying superconductivity are dual to quantum tunneling of charge destroying insulating states [16]. Excluding the former, Murani et al. [1] are expected to exclude also the latter [17]. Thus, the superconducting and the insulating states cannot exist one without the other, and they cannot disappear independently.

In their experiments, Murani et al. [1] investigate linear response at $1 \mathrm{GHz}$. Using a SQUID-loop JJ, they could tune the Josephson energy $E_{J}$ by magnetic flux. Murani et al. claim that the $\mathrm{JJ}$ is always superconducting, because they detect the effect of the magnetic flux on the response. This claim is not founded, since a nonzero supercurrent $\langle\sin \varphi(t)\rangle$ can coexist with nonzero voltage $\propto\langle d \varphi / d t\rangle$ as happens in the Bloch oscillation regime. As discussed above, a fingerprint of the insulating state is the Coulombblockade-induced resistance bump at small current bias, which can be detected only in nonlinear response. Thus, important conclusions on the DQPT can be made on the basis of measurements beyond the linear response.

Comparison with the actual predictions of DQPT theory indicate that Murani et al. [1] could not see the DQPT in their experiment. Our estimation for the bandwidth $\Delta$ of their sample 2 taking into account its renormalization by the Caldeira-Leggett effect of dissipation [see Eqs. (2.17) 
and (4.18) in Ref. [14] ] yields $\Delta \approx 0.05 \mathrm{mK}$. The insulating state is then destroyed at the electrical breakdown voltage $V_{b} \sim \Delta / 2 e$. Murani et al. [1] observe their linear response up to voltages about 1000 times larger than $V_{b}$. This observation seems possible only if all the voltage bias values are essentially above $V_{b}$. Moreover, the bandwidth $\Delta$ is smaller than the lowest measurement temperature of $10 \mathrm{mK}$, which also makes the observation of the insulating state impossible.

In summary, the analysis of Murani et al. [1] does not reveal the insulating state, because it ignores phase slips (tunnelings between minima of the phase-dependent potential) responsible for formation of the Bloch band and decay of the supercurrent. Neither the theory nor the experiment of Murani et al. [1] provides any factual grounds for the DQPT absence in a dc-probed JJ.

We thank D. Golubev, T. Heikkilä, and E. Thuneberg for discussions. This work was supported by Academy of Finland Project No. 336813 (CoE, Quantum Technology Finland), by COST Action CA16218 (NANOCOHYBRI), and also within the EU Horizon 2020 programme by ERC (QuDeT, No. 670743).

[1] A. Murani, N. Bourlet, H. le Sueur, F. Portier, C. Altimiras, D. Esteve, H. Grabert, J. Stockburger, J. Ankerhold, and P. Joyez, Absence of a Dissipative Quantum Phase Transition in Josephson Junctions, Phys. Rev. X 10, 021003 (2020).

[2] A. Schmid, Diffusion and Localization in a Dissipative Quantum System, Phys. Rev. Lett. 51, 1506 (1983).

[3] S. A. Bulgadaev, Phase Diagram of a Dissipative Quantum System, Pis'ma Zh. Eksp. Teor. Fiz. 39, 264 (1984) [JETP Lett. 39, 315 (1984)].

[4] J. S. Penttilä, U. Parts, P. J. Hakonen, M. A. Paalanen, and E. B. Sonin, "Superconductor-Insulator Transition" in a Single Josephson Junction, Phys. Rev. Lett. 82, 1004 (1999).

[5] L. S. Kuzmin, Y. V. Nazarov, D. B. Haviland, P. Delsing, and T. Claeson, Coulomb Blockade and Incoherent Tunneling of Cooper Pairs in Ultrasmall Junctions Affected by Strong Quantum Fluctuations, Phys. Rev. Lett. 67, 1161 (1991).
[6] R. Yagi, S.-i. Kobayashi, and Y. Ootuka, Phase Diagram for Superconductor-Insulator Transition in Single Small Josephson Junctions with Shunt Resistor, J. Phys. Soc. Jpn. 66, 3722 (1997).

[7] J. S. Penttilä, P. J. Hakonen, E. B. Sonin, and M. A. Paalanen, Experiments on Dissipative Dynamics of Single Josephson Junctions, J. Low Temp. Phys. 125, 89 (2001).

[8] R. K. Lindell, J. Delahaye, M. A. Sillanpää, T. T. Heikkilä, E. B. Sonin, and P. J. Hakonen, Observation of Shot-NoiseInduced Asymmetry in the Coulomb Blockaded Josephson Junction, Phys. Rev. Lett. 93, 197002 (2004).

[9] G. Liu, Y. Zhang, and C. N. Lau, Gate-Tunable Dissipation and "Superconductor-Insulator" Transition in Carbon Nanotube Josephson Junctions, Phys. Rev. Lett. 102, 016803 (2009).

[10] I. V. Pechenezhskiy, R. A. Mencia, L. B. Nguyen, Y.-H. Lin, and V. E. Manucharyan, The Superconducting Quasicharge Qubit, Nature (London) 585, 368 (2020).

[11] C. P. Herrero and A. D. Zaikin, Superconductor-Insulator Quantum Phase Transition in a Single Josephson Junction, Phys. Rev. B 65, 104516 (2002).

[12] Murani et al. [1] in their introduction use this case of unshunted JJ as an illustration of the DQPT absence. However, this case is an academic limit, since the presence of an Ohmic channel is inevitable in practice. If the shunt is absent, there is quasiparticle tunneling through the JJ, which also provides an Ohmic channel to the circuit.

[13] K. K. Likharev and A. B. Zorin, Theory of the Bloch-Wave Oscillations in Small Josephson Junctions, J. Low Temp. Phys. 59, 347 (1985).

[14] G. Schön and A. Zaikin, Quantum Coherent Effects, Phase Transitions, and the Dissipative Dynamics of Ultra Small Tunnel Junctions, Phys. Rep. 198, 237 (1990).

[15] P. Joyez, Self-Consistent Dynamics of a Josephson Junction in the Presence of an Arbitrary Environment, Phys. Rev. Lett. 110, 217003 (2013).

[16] J. E. Mooij and Y. V. Nazarov, Superconducting Nanowires as Quantum Phase-Slip Junctions, Nat. Phys. 2, 169 (2006).

[17] According to the duality principle, the environment with the $\mathrm{JJ}$ inductance added in parallel to the $\mathrm{JJ}$ at large $R_{s}$ is dual to the environment with the corresponding dual capacitance added in series with the JJ at small $R_{s}$. Then, superconductivity at small $R_{s}$ is impossible. 